\title{
ON SPHERICAL SUBMANIFOLDS WITH NULLITY
}

\author{
MARCOS DAJCZER ${ }^{1}$ AND DETLEF GROMOLL ${ }^{2}$
}

\begin{abstract}
We derive a new condition for spherical submanifolds with positive relative nullity. As an application we obtain that the Ricci curvature is bounded from above by the curvature of the ambient space.
\end{abstract}

In this note we improve earlier results of O'Neill-Stiel [O'N-S], Ferus $[\mathbf{F}]$, Abe $[\mathbf{A}]$, and Rodriguez $[\mathbf{R}]$ on complete submanifolds with positive relative nullity in euclidean spheres; cf. [R] for the basic facts and references.

THEOREM 1. Let $f: M^{n} \rightarrow S_{c}^{n+p}$ be an isometric immersion of a complete manifold in a sphere of curvature $c$ with relative nullity $\nu>0$ everywhere. Suppose there exists a point $x$ in the open set where $\nu$ is minimal so that the (normalized) Ricci curvature satisfies

$$
\operatorname{Ric}(x) \geq c
$$

Then $f$ is totally geodesic.

In $[\mathbf{A}]$ and $[\mathbf{R}]$ the same conclusion was obtained under the hypothesis that (1) holds everywhere. As an immediate consequence of our theorem, we have that if $\nu>0$ is constant and $f$ is not totally geodesic, then Ric $<c$ everywhere. Examples for this situation are the minimal (homogeneous) isoparametric hypersurfaces with three distinct principal curvatures (cf. $[\mathbf{M}]$ ). The completeness assumption above can be weakened (cf. the Remark later).

Theorem 1 will be a consequence of the following much stronger result.

THEOREM 2. Let $f: M^{n} \rightarrow S_{c}^{n+p}$ be an isometric immersion, $M^{n}$ complete, $\nu>0$ everywhere. Then the numbers of positive and negative principal curvatures in each normal direction are equal at any point where $\nu=\nu_{0}$ is minimal.

We need the following

LEMMA. Let $Q^{n+p}$ be a space of constant curvature and $f: M^{n} \rightarrow Q^{n+p}$ an isometric immersion with constant relative nullity. Then, for any parallel normal field $\xi$ of $M$ along a leaf $L$ of the nullity foliation, the rank of the second fundamental form $A_{\xi}$ is constant along $L$.

ProOF. Fix a leaf $L$. Locally in $M$, extend $\xi$, and let $V, W$ be vector fields so that $V$ is tangent and $W$ is normal to the leaves, and $W$ is parallel along $L$. By the Codazzi equations,

$$
\nabla_{V} A_{\xi} W=\nabla_{W} A_{\xi} V-A_{\nabla_{W}^{\perp} \xi} V+A_{\nabla_{V}^{1} \xi} W+A_{\xi} \nabla_{V} W-A_{\xi} \nabla_{W} V
$$

Received by the editors October 12, 1983 and, in revised form, January 9, 1984.

1980 Mathematics Subject Classification. Primary 53C40, 53B25.

${ }^{1}$ Research partially supported by CNPq, Brazil.

${ }^{2}$ Partially supported by NSF Contract MCS 8102758A02. 
where $\nabla, \nabla^{\perp}$ are the tangent and normal connections of $M$. Along $L$ the first four terms vanish. As in $[\mathbf{R}]$ we consider the tensor $B$ on the normal bundle of $L$ in $M$, where $B W$ is the normal component of $-\nabla_{W} V$. Thus, $\nabla_{V} A_{\xi}=A_{\xi} B$, or

$$
\nabla_{V} A_{\xi}=B^{t} A_{\xi}
$$

Here we view $A_{\xi}$ as being restricted to the normal bundle of $L$. By (2) $A_{\xi}$ satisfies a linear differential equation along any integral curve of $V$ in $L$, which implies that the kernel of $A_{\xi}$ is parallel and, therefore, the rank of $A_{\xi}$ is constant.

PROOF OF THEOREM 2. Consider the maximal set $U$ in $M$ where $\nu=\nu_{0}$. It is well known that (since $M$ is complete) $U$ is foliated by complete totally geodesic leaves which $f$ maps onto great spheres. Thus the antipodal map $I=-$ id of $S^{n+p}$ induces an involution $I$ on $U$. Fix $x \in U$, a normal vector $\xi$ of $M$ at $x$, and a leaf $L$ through $x$ in $U$. We can view $\xi$ as a parallel field along $L$. Note that any parallel normal field of $M$ in $S^{n+p}$ is constant along each leaf in euclidean space $\mathbf{R}^{n+p+1}$. Now we have for the second fundamental form, $I_{*} A_{\xi}=A_{I_{*} \xi} I_{*}$, so

$$
A_{\xi_{x}}=A_{-\xi_{-x}}=-A_{\xi_{-x}} .
$$

Here we identify the tangent spaces of $M$ at $x$ and $-x$ by parallel transport in euclidean space. By the Lemma $A_{\xi}$ has constant rank on the sphere $L$, so the number of positive eigenvalues of $A_{\xi_{x}}$ is equal to the number of positive eigenvalues of $A_{\xi_{-x}}$. Now our claim follows from (3).

REMARK. It is clear from the proof that the completeness of $M$ can be replaced by the weaker assumption that a leaf $L$ contains at least one pair of antipodal points. Then the conclusion of the theorem holds for all $x \in L$.

Proof of THEOREM 1. It is a simple algebraic fact that $\operatorname{Ric}(x) \geq c$ for $x \in U$ implies $A_{\xi}$ is positive definite on the normal space of the leaf $L_{x}$ in $M$ for some $\xi \neq 0$ (cf. [R, p. 183]). This contradicts Theorem 2, unless $\nu_{0}=n$, i.e. $f$ is totally geodesic.

\section{REFERENCES}

[A] K. Abe, Some remarks on a class of submanifolds in space forms of non-negative curvature, Math. Ann. 247 (1980), 275-278.

[F] D. Ferus, Isometric immersions of constant curvature manifolds, Math. Ann. 217 (1975), 155-156.

[M] R. Miyaoka, Complete hypersurfaces in the space form with three principal curvatures, Math. Z. 179 (1982), 345-354.

[O'N-S] B. O'Neill and E. Stiel, Isometric immersions of constant curvature manifolds, Michigan Math. J. 10 (1963), 335-339.

[R] L. Rodriguez, Immersions of non-zero relative nullity in manifolds of constant positive curvature, Arch. Math. 32 (1979), 181-184.

IMPA, Estrada D. CASTORINA, 110, 22460 Rio de JANEIRO, BraziL

Department of MAThematics, State University of NeW York at Stony BROOK. STONY BROOK, NEW YORK 11794 